\title{
Expérience de sélection sur la croissance du tissu maigre chez le porc, avec des pères répétés : évolution génétique des caractères soumis à la sélection
}

\author{
J Cañón ${ }^{1}, \mathrm{~J}$ Gruand ${ }^{2}$, JP Gutiérrez ${ }^{1}$, L Ollivier ${ }^{3 *}$ \\ 1 Facultad de veterinaria, departamento de producción animal, \\ 28040 Madrid, Espagne; \\ 2 Institut national de la recherche agronomique, \\ station expérimentale de sélection porcine, 86480 Rouillé; \\ ${ }^{3}$ Institut national de la recherche agronomique, station de génétique \\ quantitative et appliquée, 78352 Jouy-en-Josas, cedex, France
}

(Reçu le 28 juin 1991 ; accepté le 10 juin 1992)

Résumé - Une expérience de sélection sur la croissance du tissu maigre chez le porc Large White a été réalisée au centre d'insémination artificielle porcine de l'INRA à Rouillé (Vienne) entre 1965 et 1989. Au cours de cette période, 24 générations de verrats ont été sélectionnées sur un indice croissance-gras dorsal, et leurs descendances comparées à l'aide d'un dispositif expérimental de pères répétés. Cet article concerne l'analyse des 2 caractères mesurés sur les 2987 verrats contrôlés au cours de l'expérience : le gain moyen quotidien $(G M Q$ ) de 30 à 80 (ou 85) kg de poids vif, et l'épaisseur moyenne du lard dorsal $(L M)$ mesurée à 80 (ou 85 ) $\mathrm{kg}$ de poids vif. Un modèle mixte individuel univariate a été utilisé. Il inclut des effets de groupe génétique prenant en compte les géniteurs inconnus, c'est-à-dire les parents des pères non contrôlés (fondateurs ou immigrants) et des mères des verrats contrôlés. En fonction du sexe et de l'année de naissance de leurs produits, 35 groupes ont été définis et, après regroupement, les calculs ont été réalisés avec 27 ou 8 groupes au total. Avec le modèle à 27 groupes, les gains génétiques annuels chez les pères, sur la période $1965-1988$, sont estimés à $11,4 \mathrm{~g}$ de $G M Q$ et $-0,15 \mathrm{~mm}$ de $L M$, soit respectivement $1,4 \%$ et $0,9 \%$ de la moyenne. Les gains" génétiques annuels chez les mères, sur la période 1959-1987, sont inférieurs d'environ $20 \%$ à ceux des pères pour les 2 variables. Le modèle à 8 groupes donne des gains génétiques plus faibles, notamment pour $L M$. L'évolution génétiques de $L M$ est assez régulière sur l'ensemble de l'expérience, alors que celle du $G M Q$ est plus irrégulière, avec une tendance à un plafonnement au cours

* Correspondance et tirés à part 
des 5 dernières années. L'incidence sur les évaluations du nombre de groupes génétiques pris en considération dans le modèle est discutée, en relation avec le degré de connexion statistique du dispositif qui en résulte.

expérience de sélection / pères répétés / croissance musculaire / porc / modèle individuel

Summary - A repeat-sire mating selection experiment on lean tissue growth in the pig : genetic trends of the traits selected upon. A selection experiment on lean tissue growth in Large White pigs was carried on at the INRA artificial insemination center of Rouillé (Vienne, France) from 1965 to 1989. Over that period of time, 24 boar generations were selected on a growth-backfat index and their progeny compared using a repeat-sire mating design. The 2 traits measured on the 2987 boars tested in the experiment are analyzed in this paper, namely average daily gain ( $A D G$ ) from 30 to 80 (or 85) $\mathrm{kg}$ liveweight, and average backfat thickness (ABT) measured at 80 (or 85 ) $\mathrm{kg}$ liveweight. These traits, however, were not included in a repeat-sire design until 1977, and the design was again modified in 1984 by including male progeny from outside untested contemporary boars. The schedule of the experiment is summarized in table I, which gives the number of boars tested, selected, repeated and the number of immigrants for each year. An individual univariate mixed model has been used in the analysis. It includes genetic group effects to deal with unknown ("phantom") parents. As described in the general pedigree of figure 1, these are parents either of untested sires (founders and immigrants) or of the dams of tested boars. On the basis of the sex and year of birth of the parents' progeny, 35 genetic groups have been defined. They were regrouped in totals of 27 or 8 groups, as shown in table II, for 2 separate analyses. Under the 27-group model, the estimated sire annual genetic gains for the period 1965-1988 are $11.4 \mathrm{~g}$ and $-0.15 \mathrm{~mm}$ for $A D G$ and $A B T$ respectively, equivalent to 1.4 and $0.9 \%$ of the mean. Dam genetic trends, covering the period 1959 1987, are about $20 \%$ below sire trends for both traits. As shown in table III, the 8-group model yields lower sire and dam trends, especially for $A B T$, but dam trends are below sire trends only for $A D G$. Phenotypic trends in figure 2 and genetic trends in figures 3 and 4 show similar general patterns. Genetic trend for fatness is consistent over the whole experiment, but genetic change in growth rate is less regular and tends to plateau in the last 5 years. This may be partly due to a lower genetic merit of the immigrants, as shown in table IV. The effect of the number of genetic groups chosen in the model is discussed, in relation to the connectedness of the ensuing design.

selection experiment / repeat-sire mating / lean growth / pig / animal model

\section{INTRODUCTION}

La vitesse de croissance du tissu maigre est reconnue depuis longtemps comme un objectif essentiel de sélection chez le porc (voir, en particulier, Fowler et al, 1976). Compte tenu des évolutions génétiques récentes des populations porcines, cet objectif devrait encore être l'objet d'unc attention accrue de la part des sélectionneurs à l'avenir (Ollivier et al, 1990). Le critère généralement utilisé pour sélectionner les reproducteurs dans ce but est un indice de sélection combinant 2 caractères aisément mesurables sur l'animal vivant, la vitesse de croissance et l'épaisscur du gras dorsal. Une telle sélection a été réalisée dans une expérience conduite par l'INRA à Rouillé (Vienne) de 1965 à 1989. Des résultats partiels 
de cette expérience ont été précédemment publiés, pour la première décerınie de l'expérience (Ollivier, 1977) puis pour les 20 premières années (Ollivier, 1986). L'objet de cet article est de reprendre l'ensemble des résultats de l'expérience, qui s'est terminée en 1989 ct qui couvre donc 24 générations de verrats de race Large White sélectionnés sur un indice croissance-gras dorsal, mesuré dans des conditions d'élevage relativement constantes. Dans ce travail, nous essayons d'utiliser toute l'information disponible sur les parentés, grâce à l'utilisation d'un modèle mixtc individuel, avec constitution de groupes génétiques pour les géniteurs inconnus. Dans la première partie de cet article, nous nous limiterons aux 2 caractères mesurés sur les verrats contrôlés et servant à les sélectionner, c'est-à-dire la vitesse de croissance et l'épaisseur du gras dorsal.

\section{MATÉRIEL ET MÉTHODES}

\section{Dispositif expérimental}

Le dispositif expérimental initial et les modifications survenues au cours de l'expérience ont été précédemment décrits en détail (Ollivier, 1977 et 1986). Rappelons qu'il s'agit d'un dispositif de «pères répétés», prévu au départ pour évaluer la sélection des pères (verrats) sur des caractères $X$ et les réponses à cette sélection pour des caractères $Y$ mesurés sur des descendants (mâles castrés et femelles) abattus. De 1966 à 1976 (phase 1), les verrats contrôlés chaque année descendaient tous des verrats sélectionnés l'année précédente, et le dispositif ne prévoyait donc pas de mesurer les réponses à la sélection pour les variables $X$. Mais à partir de 1977 (phase 2) les verrats contrôlés chaque annéc descendaicnt en partic des verrats sélectionnés l'année précédente et en partie de ceux sélectionnés 2 ans auparavant. Le dispositif permettait dès lors de mesurer les réponses à la sélection pour les variables $X$ comme pour les variables $Y$. Le dispositif a de nouveau été modifié en 1984 (phase 3) en incluant parmi les verrats contrôlés chaque année des fils de verrats extérieurs à l'expérience. Ces derniers verrats (immigrants), issus d'ćlevages de sélection de race Large White appartenant à l'UPRA porcine (unité nationale de sélection et de promotion de l'espèce porcine), étaient contemporains des verrats contrôlés l'année précédente dans le cadre de l'expérience. Ils étaient sélectionnés parmi les meilleurs verrats dans les stations publiques de contrôle individuel, avec un seuil de sélection voisin de 2 écarts types au-dessus de la moyenne contemporaine de station pour l'indice de sélection utilisé dans ces stations, tel qu'il est décrit par Tibau i Font et Ollivier (1984). Le tableau I donne les effectifs des verrats contrôlés, sélectionnés et «répétés » au cours de l'expérience ainsi que les annécs de naissance des mères des verrats contrôlés.

\section{Analyse statistique}

Les variables retenues dans cette analyse sont le gain moyen quotidien à partir de $30 \mathrm{~kg}(G M Q)$ et l'épaisseur moyenne du lard dorsal au poids de fin de contrôle ( $L M)$. La période de contrôle, initialement de 30 à $80 \mathrm{~kg}$ de poids vif, a été étendue jusqu'à $85 \mathrm{~kg}$ à partir de 1977. La sćlection des verrats était basće sur un indice linéaire combinant $G M Q$ et $L M$. 
Tableau I. Verrats utilisés dans l'expérience.

\begin{tabular}{|c|c|c|c|c|c|c|c|}
\hline & \multirow{2}{*}{$\begin{array}{l}\text { Année de } \\
\text { naissance } \\
\text { des verrats }\end{array}$} & \multicolumn{5}{|c|}{ Nombre des verrats } & \multirow{2}{*}{$\begin{array}{c}\text { Année de naissance } \\
\text { des mères des } \\
\text { verrats } C\end{array}$} \\
\hline & & $C$ & $S$ & $R_{1}$ & $R_{5}$ & $I$ & \\
\hline \multirow{12}{*}{ Phase 1} & $1962-64$ & - & 10 & - & - & - & \\
\hline & 1966 & 52 & 10 & - & - & - & $1960-1965$ \\
\hline & 1967 & 50 & 10 & - & - & - & $1959-1966$ \\
\hline & 1968 & 51 & 10 & - & - & - & $1959-1967$ \\
\hline & 1969 & 73 & 11 & - & - & - & $1964-1968$ \\
\hline & 1970 & 78 & 10 & - & - & - & $1962-1969$ \\
\hline & 1971 & 97 & 8 & - & - & - & $1966-1970$ \\
\hline & 1972 & 115 & 8 & - & - & - & $1963-1971$ \\
\hline & 1973 & 99 & 8 & - & - & - & $1967-1972$ \\
\hline & 1974 & 116 & 10 & - & - & - & $1969-1973$ \\
\hline & 1975 & 119 & 8 & 5 & - & - & $1968-1974$ \\
\hline & 1976 & 104 & 10 & 7 & - & - & $1970-1975$ \\
\hline \multirow{7}{*}{ Phase 2} & 1977 & 219 & 11 & 7 & 6 & - & $1970-1976$ \\
\hline & 1978 & 234 & 11 & 9 & - & - & $1971-1977$ \\
\hline & 1979 & 148 & 10 & 9 & - & - & $1973-1978$ \\
\hline & 1980 & 195 & 7 & 7 & - & - & 1974-1979 \\
\hline & 1981 & 180 & 8 & 3 & - & - & $1976-1980$ \\
\hline & 1982 & 192 & 8 & 4 & - & - & $1976-1981$ \\
\hline & 1983 & 218 & 6 & 3 & - & 2 & $1976-1982$ \\
\hline \multirow{6}{*}{ Phase 3} & 1984 & 143 & 6 & 6 & - & 2 & $1978-1983$ \\
\hline & 1985 & 179 & 6 & 4 & - & 2 & $1978-1984$ \\
\hline & 1986 & 103 & 6 & 4 & - & 1 & $1981-1985$ \\
\hline & 1987 & 94 & 2 & 1 & - & 2 & $1983-1986$ \\
\hline & 1988 & 128 & 6 & - & - & - & $1982-1987$ \\
\hline & Total & 2987 & 200 & 68 & 6 & 9 & $\begin{array}{c}\text { 1959-1987 } \\
\text { (1 } 618 \text { mères) }\end{array}$ \\
\hline
\end{tabular}

$C$ : verrats contrôlés dans l'année $n ; S$ : verrats sélectionnés dans l'année $n ; R_{1}$ verrats $S$ répétés l'année $n+1$ (utilisation de semence fraîche); $R_{5}$ : verrats $S$ répétés l'année $n+5$ (utilisation de semence congelée); $I$ : verrats immigrants (Large White UPRA).

Les valeurs génétiques des individus mesurés dans cette expérience et de leurs parents ont été estimées par application d'un modèle linéaire incluant des effets génétiques individuels (modèle animal) et des effets de groupe génétique, selon une procédure décrite par Westell et al (1988), qui elle-même reprend un modèle initialement proposé par Thompson (1979).

Le modèle mathématique est le suivant :

$$
y_{i j}=\mu+A B_{i}+a_{j}+\Sigma_{k} p_{j k} G_{k}+c_{j}+e_{i j}
$$

où $y_{i j}$ est la variable mesuréc $(G M Q$ ou $L M) ; \mu$ la moyenne; $A B_{i}$ un effet annéebâtiment (fixé), avec $i=1, \ldots, 57 ; a_{j}$ un effet génétique additif (alćatoire) relatif 
à l'individu $j$, de variance $\sigma_{a}^{2} ; G_{k}$ un effet génétique additif fixé relatif au groupe $k$, avec $k=1, \ldots, g$ (le nombre de groupes $g$ est déterminé comme indiqué plus loin); $p_{j k}$ la contribution du groupe $k$ à la valeur génétique de l'individu $j ; c_{j}$ un effet milieu commun de la portée (aléatoire), de variance $\sigma_{c}^{2} ; e_{i j}$ un effet résiduel (aléatoire), de variance $\sigma_{e}^{2}$.

Les équations du modèle mixte obtenues à partir de [1] sont modifiées selon unc transformation décrite par Quaas et Pollak (1981) qui permet d'obtenir directement les estimées $\widehat{u}_{j}$ des valeurs génétiques individuelles:

$$
\widehat{u}_{j}=\widehat{a}_{j}+\Sigma_{k} p_{j k} g_{k}
$$

Les calculs ont été réalisés selon la méthode de Misztal et Gianola (1987). Le programme d'ordinateur nous a été aimablement fourni par I Misztal (communication personnelle). On a utilisé des valeurs a priori des héritabilités $h^{2}=\sigma_{a}^{2} /\left(\sigma_{a}^{2}+\sigma_{c}^{2}+\sigma_{e}^{2}\right)$, de 0,21 pour $G M Q$ et de 0,61 pour $L M$, et des parts de variance dues aux effets de milieu commun de la portée $\sigma_{c}^{2} /\left(\sigma_{a}^{2}+\sigma_{c}^{2}+\sigma_{e}^{2}\right)$, de 0,29 pour $G M Q$ et 0,07 pour $L M$, paramètres qui ont été estimés sur les 10 premières générations de l'expérience (Ollivier, 1983). Le critère de convergence utilisé à chaque itération $l$ est $\Sigma_{j}\left(\widehat{u}_{j}^{l+1}-\widehat{u}_{j}^{l}\right)^{2} / \Sigma_{j}\left(\widehat{u}_{j}^{l}\right)^{2}$. Une valeur-limite de $10^{-10}$ a été retenue pour l'arrêt $\mathrm{du}$ processus itératif.

Le modèle (1) permet d'estimer à la fois les valeurs génétiques des verrats, qu'ils soient contrôlés ou non (verrats fondateurs et verrats immigrants), et celles des mères des verrats contrôlés. Les réponses à la sélection sont obtenues par régression des valeurs génétiques $\widehat{u}_{j}$ en fonction de l'année de naissance des individus considérés, qui sont, du côté paternel, les verrats fondateurs et les verrats sélectionnés et immigrants ( $S$ et $I$ ) de chaque année jusqu'en 1988 et, du côté maternel, les mères des verrats contrôlés nées de 1959 à 1987. Les réponses annuelles sont calculées par régression linéaire ordinaire. Les variances des estimées $\widehat{u}_{j}$ sont calculées en faisant l'approximation d'une quantité égale d'information pour tous les verrats sélectionnés (valeur individuelle et moyenne de 11 descendants) ainsi que pour les mères (moyenne de deux descendants), sur la base des paramètres génétiques indiqués plus haut.

\section{Définition des groupes génétiques}

Suivant la définition de Westell et al (1988), nous assimilons chaque parent inconnu (parent «fantôme») à un groupe génétique, en faisant l'hypothèse que ce parent est représentatif d'un ensemble d'individus mis à la reproduction au même moment. Sont considérés comme inconnus les parents des verrats non contrôlés dans le cadre de l'expérience (verrats fondateurs et verrats UPRA) et les parents des mères des verrats contrôlés (bien qu'une certaine proportion de celles-ci soient filles des verrats de l'expérience), comme indiqué dans la figure 1. Les groupes de parents sont définis par l'année de naissance et le sexe de leurs produits. On voit d'après le tableau I que les parents des mères des verrats contrôlés se répartissent en 29 groupes, correspondant aux années de naissance 1959 à 1987, et les parents des verrats non contrôlés en 6 groupes, correspondant aux parents des verrats fondateurs (nés de 1962 à 1964 mais regroupés en une seule année de naissance) et des verrats 


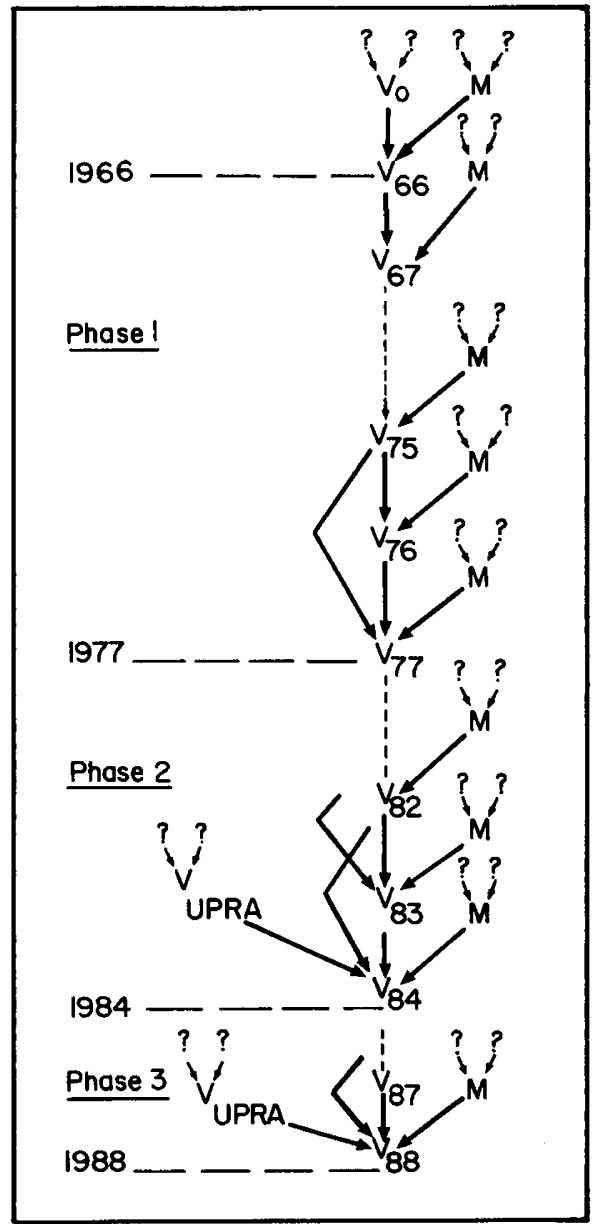

Fig 1. Pedigree des animaux au cours des 3 phases de l'expérience. $V_{0}:$ verrats fondateurs (Herd-Book Large White); $V_{66}$ : verrats contrôlés en 1966, $V_{67}$ : verrats contrôlés en 1967 , etc...; $M$ : mères des verrats contrôlés (population des truies inséminées); $V_{\text {UPRA }}$ verrats Large White non contrôlés dans l'expérience (origine UPRA);? : individus sans identification.

immigrants $(I)$ nés de 1983 à 1987, soit un total $g=35$. A titre d'exemple, la contribution des groupes ainsi définis à la valeur génétique d'un verrat contrôlé en 1967, issu de grands parents paternels nés tous 2 en 1964 et d'une mère née en 1965 est égale à $0,25 G_{0}+0,25 G_{64}+0,5 G_{65}$. Les pères et mères des verrats fondateurs $\left(V_{0}\right)$ nés en 1964 sont ainsi considérés comme appartenant à un groupe génétique $\left(G_{0}\right)$ différent de celui des parents des mères $(M)$ nées en $1964\left(G_{64}\right)$, car $V_{0}$ et $M$ sont en fait issus de populations différentes. Mais il faut remarquer que les 
descendants des verrats fondateurs ont tous ćté contrôlés la même année et dans le même bâtiment, ce qui induit une confusion statistique entre les niveaux $A B_{1}$ et $G_{1}$ du modèle (1), confusion qui n'existe pas pour les autres niveaux. Pour y remédier, un groupe génétique unique de fondateurs (verrats $V_{0}$ et mères $M$ ) a dû être constitué. En outre, les groupes définis plus haut contribuent très inégalement à l'ensemble des individus contrôlés, ce qui justific aussi des regroupements. Comme indiqué au tableau II, le nombre total de groupes a ainsi été ramené à $g=27$ ou $g=8$.

Tableau II. Constitution des groupes génétiques.

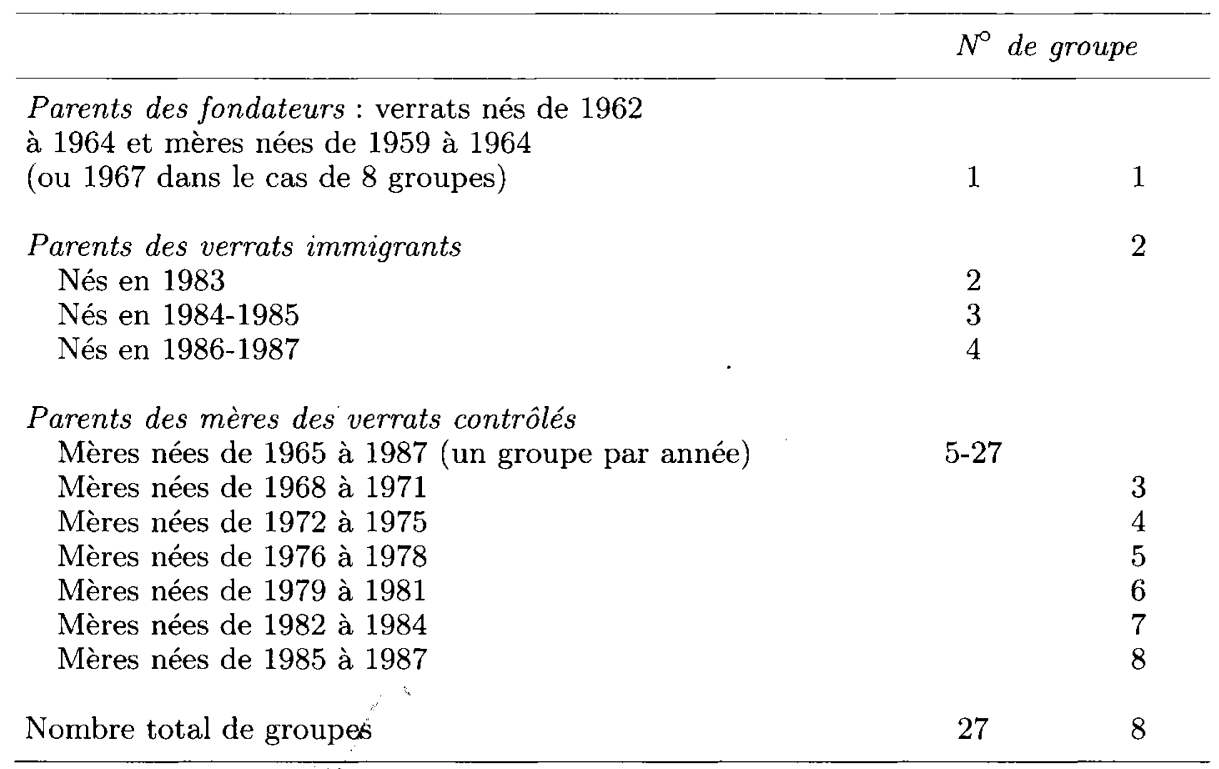

\section{RÉSULTATS}

L'évolution des moyennes phénotypiques annuelles est donnée dans la figure 2. L'évolution des valeurs génétiques moyennes annuelles est représentée dans les figures 3 et 4 , respectivement pour les pères sélectionnés chaque année, de 1965 (verrats fondateurs, regroupés dans l'année 1965) à 1988, et pour les mères des verrats contrôlés, nées de 1959 à 1987. On note que l'évolution génétique des pères est surtout marquée sur la période 1971-1982 pour la vitesse de croissance (GMQ), alors que l'épaisseur du gras dorsal $(L M)$ suit une évolution plus régulière.

Les tendances génétiques linéaires sont données au tableau III. Avec le modèle à 27 groupes, les tendances maternelles sont inféricures aux tendances paternelles de l'ordre de $20 \%$ pour les 2 caractères. Avec le modèle à 8 groupes, les tendances patcrnelles et maternelles sont plus faibles qu'avec 27 groupes, notamment pour $L M$ et la tendance maternelle n'est inférieure à la tendance paternelle que pour $G M Q$. 

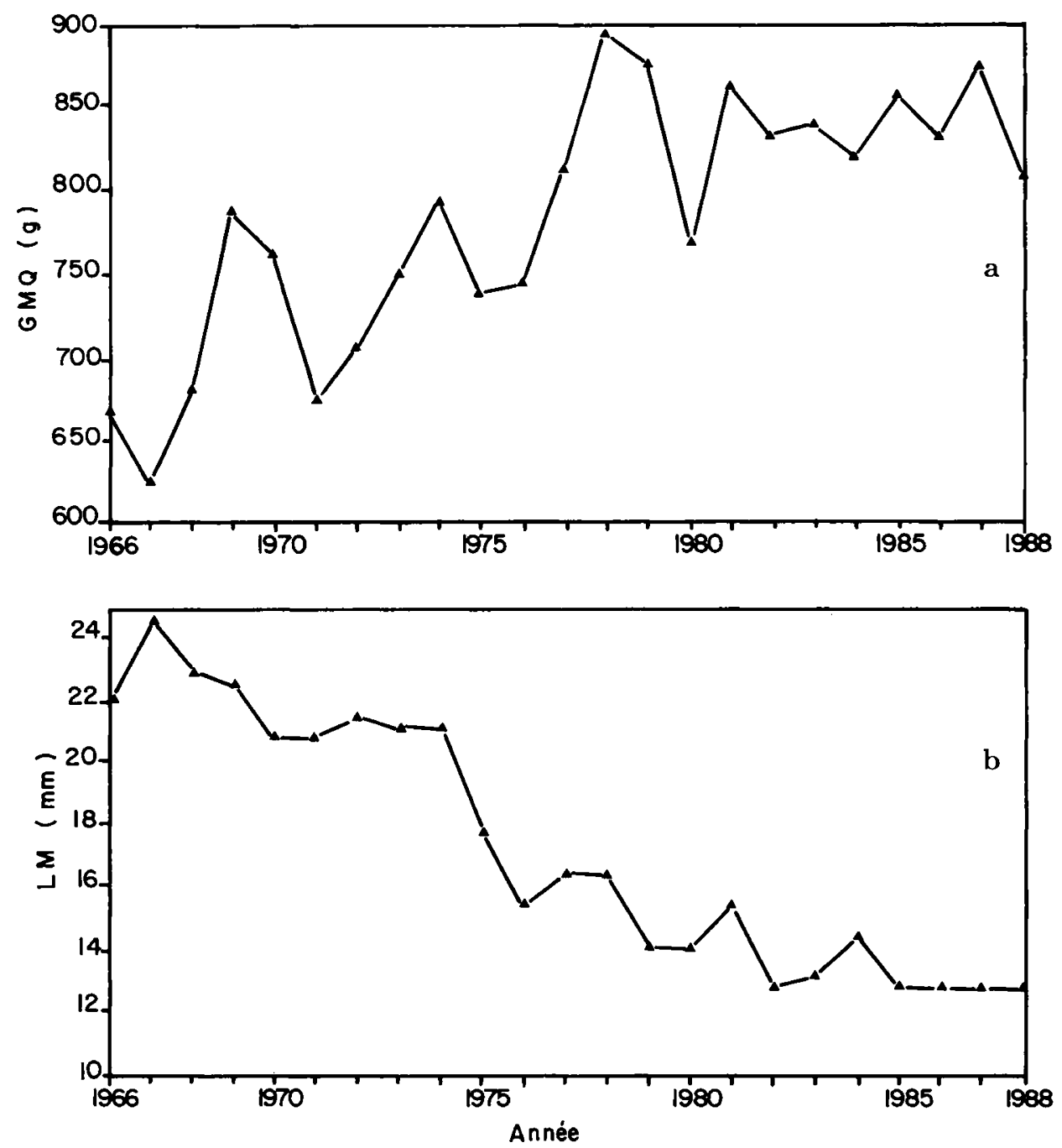

Fig 2. Évolutions phénotypiques : a, de la vitesse de croissance $(G M Q)$; b, de l'épaisseur du lard dorsal (LM).

L'analyse permet aussi de comparer le niveau génétique des verrats introduits de l'extérieur, de 1984 à 1987, à celui des verrats sélectionnés dans l'expérience. Les résultats du tableau IV montrent que les verrats immigrants croissent moins vite et sont moins gras que les verrats de l'expérience. 

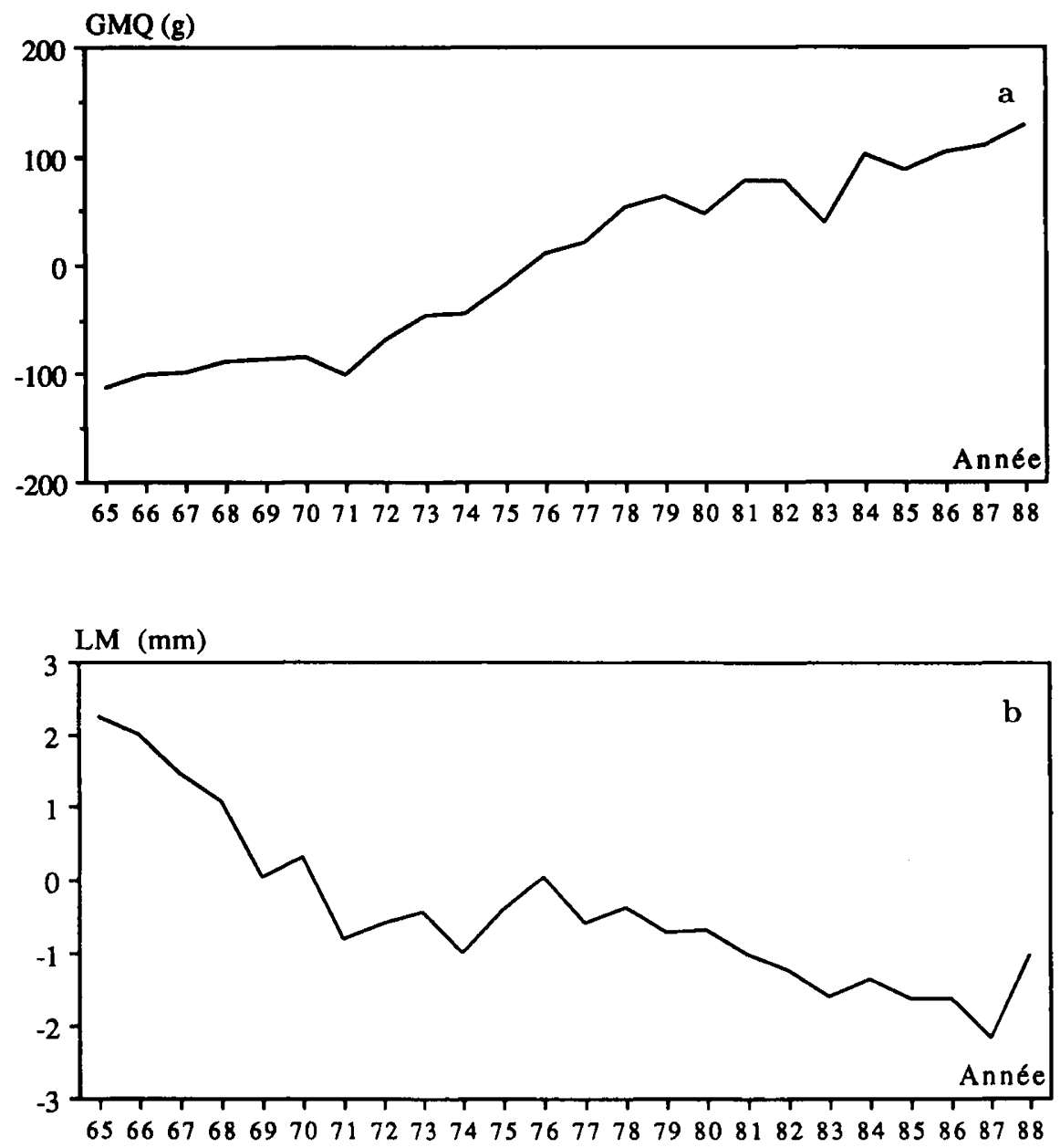

Fig 3. Évolutions génétiques paternelles (modèle à 27 groupes génétiques) : a, de la vitesse de croissance $(G M Q)$; b, de l'épaisseur du lard dorsal $(L M)$.

\section{DISCUSSION ET CONCLUSION}

Le dispositif expérimental d'accouplements répétés (Goodwin et al, 1955) et sa version simplifiée par répétition des pères seulement (Dickerson, 1960; Hickman et Freeman, 1969) ont été peu utilisés jusqu'à présent pour l'évaluation des réponses à la sélection. A côté de l'expérience analysée ici, qui semble être la seule de ce type pour les animaux de ferme, il faut mentionner que le dispositif a aussi été expérimenté sur animaux de laboratoire (Parker et al, 1980).

Plusieurs méthodes s'offrent pour estimer les réponses à la sélection dans un tel dispositif. La méthode la plus simple consiste à exploiter directement le dispositif, 

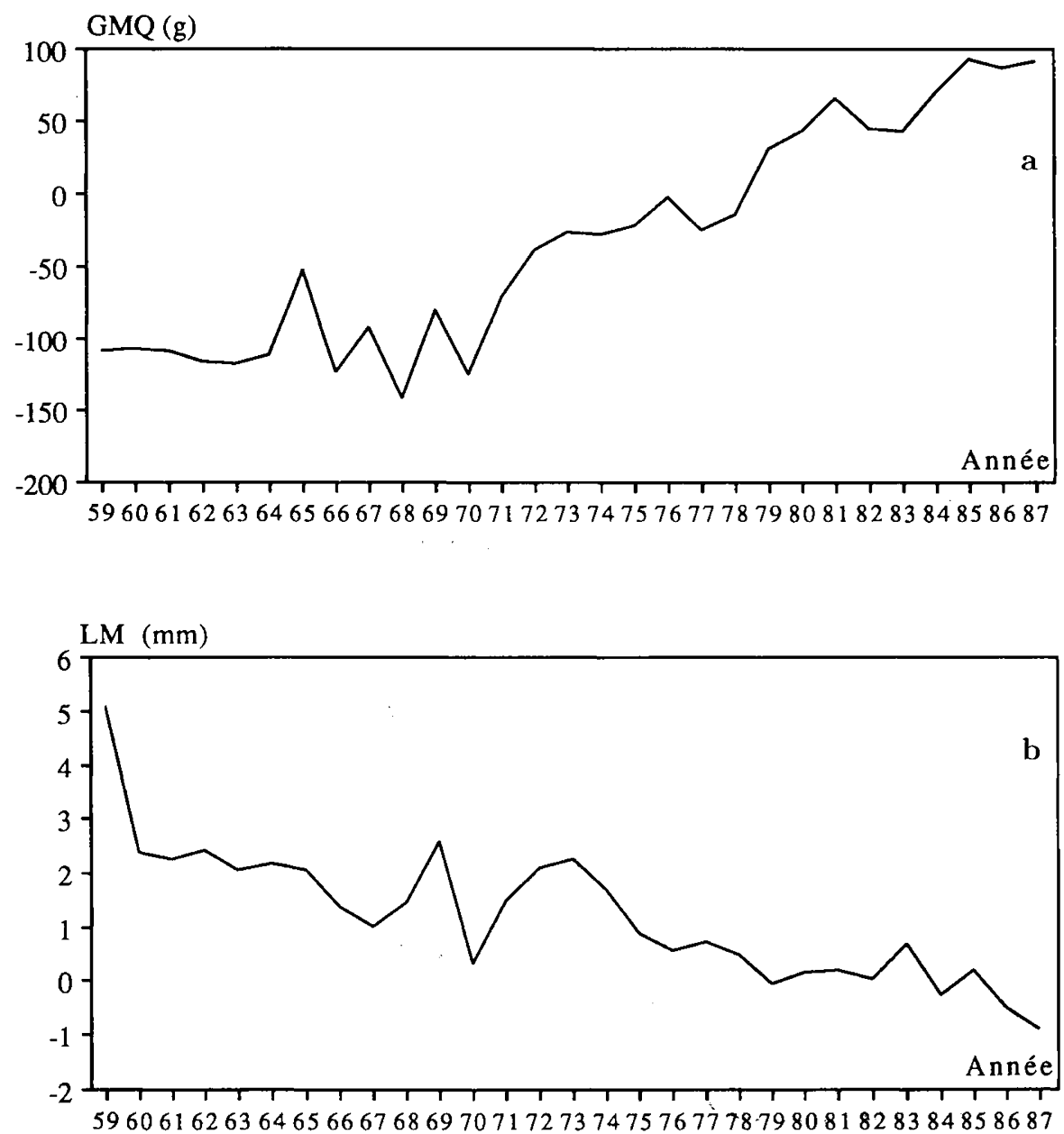

Fig 4. Évolutions génétiques maternelles (modèle à 27 groupes génétiques) : a, de la vitesse de croissance $(G M Q) ; \mathbf{b}$, de l'épaisseur du lard dorsal $(L M)$.

puisqu'il est conçu pour dissocier les effets génétiques (générations ou cohortes successives) des effets de milieu par une simple analyse de moindres carrés. C'est la méthode qui a servi à analyser la première décennie de cette expérience (Ollivier, 1977). L'application d'un modèle "mixte» paternel, par opposition au modèle «fixé» précédent, offre l'avantage d'estimer les valeurs génétiques individuelles des pères utilisćs, et ainsi d'éliminer d'ćventucls biais consécutifs à la non répétition de certains pères. C'est la méthode qui a été appliquée à l'analyse des 20 premières générations (Ollivier, 1986). L'utilisation d'un modèle mixte individuel (ou modèle animal), appliqué ici à l'ensemble de l'expérience, offre l'avantage d'estimer la valeur génétique de chaque individu contrôlé sur la base de sa valeur phénotypique et de 
Tableau III. Moyennes générales et tendances phénotypiques et génétiques annuelles.

\begin{tabular}{|c|c|c|}
\hline & $G M Q(g)$ & $L M(m m)$ \\
\hline Moyenne générale $(n=2987)$ & 803 & 16,42 \\
\hline Ecart-type résiduel (intra-bâtiment) & 110 & 2,16 \\
\hline Tendance phénotypique & $7,9 \pm 0,39$ & $-0,57 \pm 0,008$ \\
\hline \multicolumn{3}{|c|}{ Tendances génétiques avec le modèle à 27 groupes } \\
\hline Pères & $11,4 \pm 0,53$ & $-0,15 \pm 0,018$ \\
\hline Mères & $9,5 \pm 0,22$ & $-0,12 \pm 0,010$ \\
\hline \multicolumn{3}{|c|}{ Tendances génétiques avec le modèle à 8 groupes } \\
\hline Pères & $9,0 \pm 0,51$ & $-0,05 \pm 0,018$ \\
\hline Mères & $7,0 \pm 0,23$ & $-0,05 \pm 0,010$ \\
\hline
\end{tabular}

$G M Q$ : vitesse de croissance de 30 à $80 \mathrm{~kg}$ (85 kg à partir de 1977); $L M$ : épaisseur moyenne du lard dorsal à $80 \mathrm{~kg}(85 \mathrm{~kg}$ à partir de 1977$)$.

Tableau IV. Valeurs génétiques moyennes des verrats sélectionnés ( $S$ ) dans l'expérience de 1983 à 1987 et des verrats contemporains immigrants (I).

\begin{tabular}{lrrrrr}
\hline \multirow{2}{*}{ Verrats } & \multicolumn{2}{c}{$G M Q(\mathrm{~g})$} & & \multicolumn{2}{c}{$L M(\mathrm{~mm})$} \\
\cline { 2 - 3 } \cline { 5 - 6 } & 27 groupes & 8 groupes & & 27 groupes & 8 groupes \\
\hline Sélectionnés (26) & 95 & 76 & & $-1,32$ & $-1,10$ \\
Immigrants (9) & 55 & 51 & & $-2,51$ & $-2,23$ \\
Différence S-I & $40 \pm 33$ & $25 \pm 19$ & & $1,19 \pm 0,47$ & $1,13 \pm 0,47$ \\
\hline
\end{tabular}

Variables $G M Q$ et $L M$ définies au tableau III.

celle de tous ses apparentés, après élimination des effets de milieu. Les générations successives sont ainsi connectées entre elles à travers les pedigrees et un dispositif de répétitions d'accouplements n'est plus alors strictement nécessaire. Cela a permis en fait dans notre analyse d'évaluer les valeurs génétiques relatives de l'ensemble des 24 cohortes de verrats (1965-1988), alors que l'analyse précédente (Ollivier, 1986) ne permettait pas de remonter avant 1975.

En contrepartie, l'utilisation d'un modèle mixte implique un modèle génétique sous-jacent, avec des paramètres génétiques supposés connus. Nous avons choisi un modèle univariate, avec 2 paramètres $\sigma_{a}^{2}$ et $\sigma_{c}^{2}$ pour chaque caractère, de préférence à un modèle bivariate qui aurait impliqué 6 paramètres et une corrélation génétique pour laquelle des estimations assez peu concordantes avaient été obtenues sur les 10 premières générations de l'expérience (voir Ollivier, 1983, p 108). L'intérêt des modèles mixtes dans l'analyse des cxpériences de sćlection a été discuté en détail par Sorensen et Kennedy (1986) et Kennedy (1990). Soulignons la nécessité, en l'absence de témoin, d'un recouvrement suffisant des générations successives, pour obtenir des estimations qui ne soient pas trop dépendantes du modèle utilisé (Thompson, 1986). 
Notons que dans notre expérience, avant 1975, il y avait sculement recouvrement des générations maternelles.

La prise en compte d'effets de groupes génétiques, tels que nous les avons définis dans l'analyse du modèle (1). est justifiéc par la structure de l'information recucillic dans cette expérience de sélection. Contrairement aux expériences de sélection classiques, celle-ci ne permettait pas de contrôler la totalité de la sélection pratiquée. du fait des immigrations (voir Ollivier, 1985). La population considérée est en effet ouverte à des migrations de géniteurs issus de populations extérieures à l'expérience et soumises à des pressions de sélection qui leur sont propres et qui sont souvent inconnues. Tel est le cas d'une part des mères des verrats contrôlés dans leur majorité, depuis le début de l'expérience, et d'autre part des verrats immigrants introduits à partir de 1983. Ignorer l'existence de groupes dans ce contexte reviendrait à considérer que tous les individus sans pedigree (les inconnus de la figure 1) apparticnnent à une même population de base. Ainsi, par exemple, les mères nées en 1987 seraient alors supposées provenir de la même population que les mères nées en 1960. Comme indiqué par Westell et al (1988), les effets de groupe rendent compte d'une sélection qui n'apparait pas dans les données, à travers des rclations de parenté connues.

Le problème pratique qui surgit alors est de décider du nombre de groupes à prendre en compte et de la manière de les constituer. C'est unc question très débattue et à laquelle on ne peut pas pour le moment donner de réponse précise et suffisamment générale. Un risque à éviter est celui, souligné par Quaas (1988), des confusions statistiques qui peuvent se présenter dans l'estimation des effets de groupe, si leur nombre est trop élevé. La question rejoint le problème plus général de la «connexion» statistique dans les dispositifs d'évaluation génétique, qui a été récemment discuté de manière approfondie par Foulley et al (1990). La variance de l'erreur de prédiction augmente en effet dans les dispositifs à groupes nombreux et mal connectés, mais, à l'opposé, en réduisant le nombre des groupes pour améliorer les connexions on se heurte au risque de biais des évaluations. Dans notre cas, l'évaluation du niveau génétique des fondatcurs est exposée à ce risque, puisque, comme on l'a vu, le dispositif expérimental impose de regrouper les fondateurs mâles et femelles en un groupe unique. Nous avons, par ailleurs, choisi de comparer 2 stratégies de groupcment dans notre analyse, avec d'une part un nombre total de groupes (27) proche du maximum possible en se basant sur les années de naissance, et d'autre part une réduction de ce nombre à 8 en rassemblant plusicurs années dans un même groupe. A défaut de critères précis du degré de connexion tels que ceux décrits par Foulley et al (1990) ou la mesure proposée récemment par Foulley et al (1992). le nombre des itérations nécessaires pour parvenir aux solutions pourrait constitucr un indicateur de la valeur relative des 2 dispositifs que nous avons constitués. Il apparait cn effet que le nombre des itérations est considérablement diminué (de 26 et $53 \%$ pour $G M Q$ et $L M$ respectivement) quand on passe de 27 à 8 groupes. En contrepartic, la réduction du nombre des groupes génétiques à 8 tend à surestimer le niveau génétique des parents des fondateurs et à sous-estimer cclui des mères en fin d'expérience. Il en résulte un tassement des valeurs génétiques extrêmes, que montrent les évolutions annuclles, et qui entraîne une sous-estimation des évolutions génétiques. On peut également penser que le degré de connexion a ćtć amélioré à partir de 1977 avec l'extension du dispositif des pères répétés aux 
variables $G M Q$ et $L M$. Une analyse séparée de la période 1966-1976 (phase 1) et de la période 1977-1988 (phases 2 et 3) montre que la vitesse de convergence n est que faiblement ralentie dans cette dernière période malgré le doublement du nombre des équations.

En conclusion. si on retient le modèle à 27 groupes, qui minimisc le risque de biais, l'efficacité de la sélection se traduit par des gains génétiques annuels moyens de $1.4(11,4 \mathrm{~g})$ et $0,9(-0,15 \mathrm{~mm}) \%$ de la moyenne, respectivement pour $G M Q$ et $L M$. Sur les 24 générations de verrats de cette expérience, le gain génétique total pour GMQ est d'environ $240 \mathrm{~g}$ et la réduction de l'épaisseur du tissu adipeux dorsal sous-cutané est d'environ $4 \mathrm{~mm}$. Le gain phénotypique total observé pour $G M Q$ (fig 2), d'environ $200 \mathrm{~g}$, est inférieur au gain génétique, malgré le passage d'une alimentation restreinte à une alimentation à volonté des verrats en 1969 et l'augmentation de 80 à $85 \mathrm{~kg}$ du poids de fin de contrôle en 1977. Il faut en revanche noter que la réduction totale de l'adiposité dorsale est nettement plus marquée phénotypiquement (environ $10 \mathrm{~mm}$ ) que génétiquement, et ce malgré l'augmentation de $5 \mathrm{~kg}$ du poids vif au moment de la mesure (intervenue en 1977), ce qui équivaut à environ $1 \mathrm{~mm}$ de plus. Cette différence pourrait provenir d'un changement dans la méthode de mesure du gras dorsal, qui s'est traduit par une diminution de plus de $5 \mathrm{~mm}$ entre 1974 et 1976 (voir fig 2b). Alors que l'évolution génétique de l'adiposité des verrats est assez régulière tout au long de l'expérience, un plafonnement de la vitesse de croissance à partir de 1984 est à noter. L'introduction de verrats extérieurs, de niveau génétique inférieur en croissance à celui des verrats sélectionnés dans l'expérience (tableau IV). pourrait ne pas être étrangère à l'infléchissement observé. L'analyse de mesures plus détaillées recucillies sur les descendants des verrats considérés dans cet article devrait permettre de préciser ces évolutions génétiques.

\section{REMERCIEMENTS}

Les auteurs remercient $\mathrm{F}$ Bariteau et $\mathrm{J}$ Bussières (Station expérimentale d'insémination artificielle de Rouillé) pour le soin apporté à l'utilisation des verrats sélectionnés et sont reconnaissants à $\mathrm{H}$ Bernardin, C Felgines, M Joseph et $\mathrm{H}$ Lagant pour leur contribution au traitement informatique des données.

Ce travail a bénéficié d'une aide accordée dans le cadre d'une action intégrée francoespagnole cofinancée par le ministère espagnol de l'Education et de la Science et le ministère français des Affaires étrangères.

\section{RÉFÉRENCES}

Dickerson GE (1960) Technique for research in quantitative animal genetics. In : Techniques and procedures in animal production research. Am Soc Anim Prod. Beltsville, MD, 56-105.

Foulley JL, Bouix J, Goffinet B, Elsen JM (1990) Connectedness in genetic evaluation. In : Advances in statistical methods for genetic improvement of livestock (D Gianola, K Hammond, eds) Springer-Verlag. Berlin. 277-308

Foulley JL, Hanocq E, Boichard D (1992) A criterion for measuring the degree of connectedness in linear models of genetic evaluation. Genet Sel Evol (sous pressc) 
Fowler VR, Bichard M, Pease A (1976) Objectives in pig breeding. Anim Prod 23, 365-387

Goodwin K, Dickerson GE, Lamoreux WF (1955) A technique for measuring genetic progress in poultry breeding experiments. Poult Sci 38, 1197

Hickman CG, Freeman AE (1969) New approach to experimental designs for selection studies in dairy cattle and other species. J Dairy Sci 52, 1044-1054

Kennedy BW (1990) Use of mixed model methodology in analysis of designed experiments. In : Advances in statistical methods for genetic improvement of livestock (D Gianola, K Hammond, eds) Springer-Verlag, Berlin, 77-97

Misztal I, Gianola D (1987) Indirect solution of mixed model equations. J Dairy Sci 70, 716-723

Ollivier L (1977) Dix ans d'une expćrience de sélection individuelle sur des verrats utilisés en insémination artificielle. I. Réponses observécs sur des caractères de croissance, de carcasse et de qualité de viande. Ann Génét Sél Anim 9, 353-377

Ollivier L (1983) Dix ans d'une expérience de sélection individuelle sur des verrats utilisés en insémination artificielle. II. Paramètres génétiques estimés. Génét Sél Evol 15, 99-198

Ollivier L (1985) Dix ans d'une expérience de sélection individuelle sur des verrats utilisés en insémination artificielle. III. Paramètres génétiques réalisés. Génét Sél Evol 17, 481-498

Ollivier L (1986) Results of a long-term selection experiment for lean tissue growth in the pig. In : 3rd world congress on genetics applied to livestock production (GE Dickerson, RK Johnson, eds) Univ Lincoln, Lincoln, NE, vol 12, 168-189

Ollivier L, Guéblez R, Webb AJ, van der Steen HAM (1990) Breeding goals for nationally and internationally operating pig breeding organisations. In : 4th world congress on genetics applied to livestock production (WG Hill, R Thompson, JA Woolliams, eds) Midlothian, Scotland, vol 15, 383-394

Parker RJ, Cheung TK, Hickman CG, Wittenberg KM (1980) A repeat mating design in a selection experiment. Can J Anim Sci 60, 873-841

Quaas RL (1988) Additive genetic models with groups and relationships. $J$ Dairy Sci $71,1338-1345$

Quaas RL, Pollak EJ (1981) Modified equations for sire models with groups. $J$ Dairy Sci 64, 1868-1872

Sorensen D, Kennedy BW (1986) Analysis of selection experiments using mixed model methodology. J Anim Sci 68, 245-258

Thompson R (1979) Sire evaluation. Biometrics 35, 339-353

Thompson R (1986) Estimation of realized heritability in a selected population using mixed models methods. Genet Sel Evol 18, 475-484

Tibau i Font J, Ollivier L (1984) La sélection en station chez le porc. Bull Tech Dép Génét Anim INRA n 37, 69 p

Westell RA, Quaas RL, Van Vleck LD (1988) Genetic groups in an animal model. J Dairy Sci 71, 1310-1318 